\title{
Quadrotor UAV trajectory tracking based on iterative self learning control
}

\author{
YANG Li-ben*, ZHANG Wei-guo, HUANG De-gang \\ Department of Automatic Control, Northwestren Polytechnical University, Xi'an 710129,China \\ *yangliben0880@163.com
}

Keywords: Quadrotor UAV; self-learning control; Iterative correction; Trajectory tracking

\begin{abstract}
In order to solve the problems in the traditional control method of Quadrotor UAV, Such as weak anti-interference ability and system modeling error has a greater impact on the tracking performance, A trajectory tracking algorithm based on self-learning control is designed. The algorithm can control the object accurately by the iterative correction. To control the unknown object in a certain time, have good anti-interference ability, It has good control effect for the uncertain system and nonlinear coupling system, As the structure of the control algorithm is relatively simple, So it is easy to implement in actual system. The simulation results verify the feasibility of the algorithm.
\end{abstract}

\section{Introduction}

The Quadrotor UAV with 4 separate motor drive, Pitch and roll motion is achieved by differential torque, The yaw motion is achieved by anti-torque of rotors, Total lift is generated for 4 rotors, Due to the instability of its structure, the Quadrotor UAV Need real-time attitude control[1].

At present, the research of Quadrotor UAV is mainly concentrated on the aspects of robust attitude and trajectory control, The controller of the Quadrotor UAV was designed based on PID, fuzzy control, LQ control, loop shaping theory[2-5], The paper[6-7] designs a feedback linearization controller for the Quadrotor UAV.The modeling error has great influence on the feedback linearization control, The control effect is not satisfactory when the system is disturbed, The controller was designed by backstepping method in the reference [8-9], backstepping control is also susceptible to modeling errors.

The controller is designed by the dynamic surface method in the literature [10], The dynamic surface method is used to reduce the order of the derivative in the backstepping control, The controller is designed by sliding mode control in the literature [11-12].

The above method can increase the robustness of flight control system,But the modeling error has great influence on the control effect. And the design process of controller is complex. So I designed a kind of $r$ trajectory control method based on iterative self-learning control. The method does not depend on the exact mathematical model of the system, The control input is changes with the previous control input and tracking error. The control accuracy can be improved continuously in control process.

\section{Quadrotor UAV mathematical model}

Choose the body coordinate system to describe the motion of the aircraft, The gravity of the body is coincident with the origin of the coordinates, The structure of the Quadrotor UAV is shown in Figure 1. 


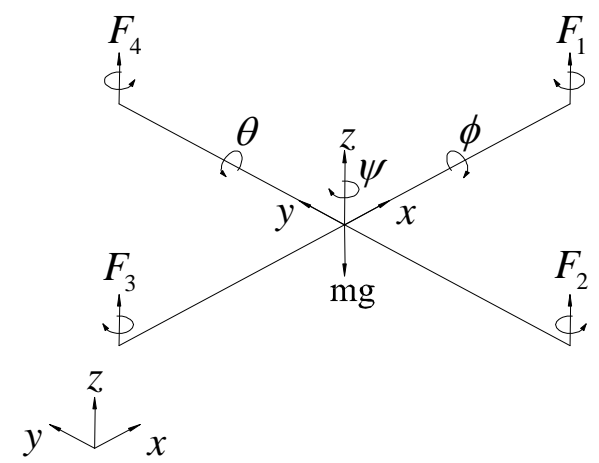

Fig. 1 Flying principle of the quadrotor UAV

According to the structure of the system, The nonlinear mathematical model of the Quadrotor UAV is

$$
\left\{\begin{array}{l}
\dot{\xi}=v \\
m \dot{v}=-e_{3} m g+R F \\
\dot{\eta}=W(\eta) \lambda \\
J \dot{\lambda}=-\lambda \times J \lambda+\tau
\end{array}\right.
$$

Among them, $\xi$ is the position vector, $v$ is the speed vector, $e_{3}=(0,0,1)^{T}, F$ is the total external force vector, $R$ is the conversion matrix From the body coordinate system to the ground coordinate system[14], $\eta$ is the euler angle of UAV, $\lambda$ is angular speed of UAV, $W(\eta)$ is the conversion matrix of the three axis angular velocity in the body coordinate system to the euler angular rate, $J$ is the inertia matrix for UAV, $\tau$ is the Control torque of aircraft.

The control input is shown in formula 2 and 3.

$$
\begin{aligned}
& F=\left[\begin{array}{l}
0 \\
0 \\
T
\end{array}\right]=\left[\begin{array}{c}
0 \\
0 \\
b_{r} \sum_{i=1}^{4} \omega_{i}^{2}
\end{array}\right] \\
& \tau=\left[\begin{array}{c}
\tau_{\phi} \\
\tau_{\theta} \\
\tau_{\psi}
\end{array}\right]=\left[\begin{array}{c}
l b_{r} \rho A\left(\omega_{2}^{2}-\omega_{4}^{2}\right) \\
l b_{r} \rho A\left(\omega_{1}^{2}-\omega_{3}^{2}\right) \\
b_{q} \rho A\left(\omega_{2}^{2}+\omega_{4}^{2}-\omega_{1}^{2}-\omega_{3}^{2}\right)
\end{array}\right]
\end{aligned}
$$

Among them, $b_{r}$ is the thrust coefficient of the rotor, $b_{q}$ is the Anti torque coefficient of the rotor, $\rho$ represents air density, $A$ is the rotor rotation area, Motor speed of four motors are respectively $\omega_{i}(i=1 . .4)$.

The system state variables defined for the Quadrotor UAV are as follows

$$
X=[x \dot{x} \theta \dot{\theta} y \dot{y} \phi \dot{\phi} z \dot{z} \psi \dot{\psi}]
$$

The state $x$ and $y$ belong to the indirect driving state, The state $\theta 、 \phi 、 \psi$ and $z$ belong to the direct driving state, The structure of trajectory control is as follows. 


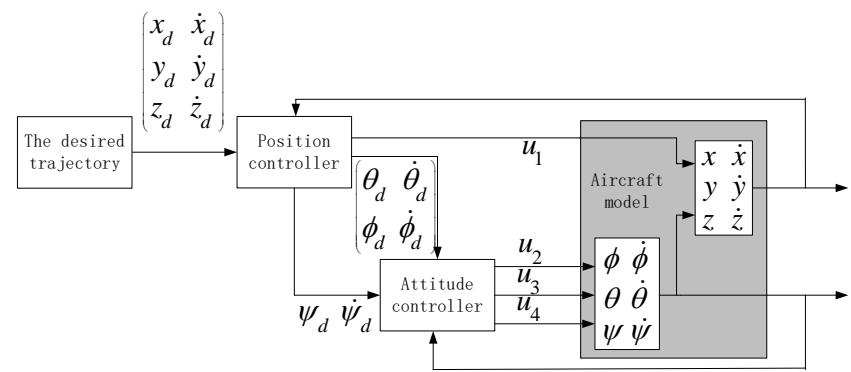

Fig. 2 Structure diagram of quadrotor aircraft trajectory control system

\section{Iterative self learning trajectory control}

The iterative self learning control is accomplished by the iterative correction of the control object. It can control the unknown object in a certain time. It has the low requirement for the accuracy of the control system modeling. It is a kind of intelligent control. And have strict mathematical proof, It does not depend on the mathematical model of the system, It has good control effect for the uncertain system and nonlinear coupling system.

In this paper, the trajectory control of Quadrotor UAV is realized by the iterative self-learning control, According to the formula (1), the trajectory equation of the aircraft can be got as follows.

$$
\left\{\begin{array}{l}
\ddot{x}=(\sin \psi \sin \phi+\cos \psi \sin \theta \cos \phi) T / m+\sigma_{x} \\
\ddot{y}=(-\cos \psi \sin \phi+\sin \psi \sin \theta \cos \phi) T / m+\sigma_{y} \\
\ddot{z}=(\cos \phi \cos \theta) \frac{T}{m}-g+\sigma_{z}
\end{array}\right.
$$

In the formula, $\sigma_{i}(i=x, y, z)$ is the compound interference of each channel, Including system modeling error and outside interference, The virtual control of the horizontal direction is as follows.

$\left\{\begin{array}{l}u_{x}=\sin \psi \sin \phi+\cos \psi \sin \theta \cos \phi \\ u_{y}=-\cos \psi \sin \phi+\sin \psi \sin \theta \cos \phi\end{array}\right.$

Set the expected trajectory and its derivatives, respectively $x_{d}, \dot{x}_{d}, y_{d}, \dot{y}_{d}$ and $z_{d} 、 \dot{z}_{d}$, The iterative learning control algorithm is as follows.

$$
\left\{\begin{array}{l}
u_{x}=k_{p}^{x} \tilde{\lambda}_{x}+k_{d}^{x} \dot{\tilde{\lambda}}_{x}+f\left(x, \dot{x}, \dot{\tilde{\lambda}}_{x}\right) \gamma_{x} \\
\gamma_{x}=\gamma_{x}+H_{x} f\left(x, \dot{x}, \dot{\tilde{\lambda}}_{x}\right) \dot{\tilde{\lambda}}_{x}
\end{array}\right.
$$

In the formula, $\tilde{\lambda}_{\mathrm{k}}^{x}=x_{d}-x_{k}, \quad \dot{\tilde{\lambda}}_{x}=\dot{x}_{d}-\dot{x}, f\left(x, \dot{x}_{x}\right)=k_{\lambda}^{x} \operatorname{sgn}\left(\dot{\tilde{\lambda}}_{x}\right)\left(k_{\lambda}^{x}>0\right), H_{x}$ is a positive definite symmetric matrix.

$$
\left\{\begin{array}{l}
u_{y}=k_{p}^{y} \tilde{\lambda}_{y}+k_{d}^{y} \dot{\tilde{\lambda}}_{y}+f\left(y, \dot{y}, \dot{\tilde{\lambda}}_{y}\right) \gamma_{y} \\
\gamma_{y}=\gamma_{y}+H_{y} f\left(y, \dot{y}, \dot{\tilde{\lambda}}_{y}\right) \dot{\tilde{\lambda}}_{y}
\end{array}\right.
$$

In the formula, $\tilde{\lambda}_{y}=y_{d}-y, \quad \dot{\tilde{\lambda}}_{y}=\dot{y}_{d}-\dot{y}, \quad f\left(y, \dot{y}, \dot{\tilde{\lambda}}_{y}\right)=k_{\lambda}^{y} \operatorname{sgn}\left(\dot{\tilde{\lambda}}_{y}\right)\left(k_{\lambda}^{y}>0\right), H_{y}$ is a positive definite symmetric matrix.

$$
\left\{\begin{array}{l}
T=k_{p}^{z} \tilde{\lambda}_{z}+k_{d}^{z} \dot{\tilde{\lambda}}_{z}+f\left(z, \dot{z}, \dot{\tilde{\lambda}}_{z}\right) \gamma_{z} \\
\gamma_{z}=\gamma_{z}+H_{z} f\left(z, \dot{z}, \dot{\tilde{\lambda}}_{x}\right) \dot{\tilde{\lambda}}_{z}
\end{array}\right.
$$


In the formula , $\tilde{\lambda}_{z}=z_{d}-z, \quad \dot{\tilde{\lambda}}_{z}=\dot{z}_{d}-\dot{z}, \quad f\left(z, \dot{z}, \dot{\tilde{\lambda}}_{z}\right)=k_{\lambda}^{z} \operatorname{sgn}\left(\dot{\tilde{\lambda}}_{z}\right)\left(k_{\lambda}^{z}>0\right), H_{z}$ is a positive definite symmetric matrix.

\section{Algorithm simulation}

To verify the effectiveness of the trajectory tracking algorithm in this paper. The simulation of circular trajectory tracking is carried out, The parameters of the Quadrotor UAV are as follows, $m=0.75 \mathrm{~kg}, l=0.25 \mathrm{~m}$, $k_{t}=3.13 \mathrm{e}^{-5} \mathrm{Ns}^{2},, k_{d}=7.5 \mathrm{e}^{-7} \mathrm{Nms}^{2}, I_{x}=19.688 \mathrm{e}^{-3} \mathrm{kgm}^{2}, I_{y}=19.681 \mathrm{e}^{-3} \mathrm{kgm}^{2}$, $I_{z}=3.938 \mathrm{e}^{-2} \mathrm{kgm}^{2}, J_{\text {rotor }}=6 \mathrm{e}^{-5} \mathrm{kgm}^{2}$.

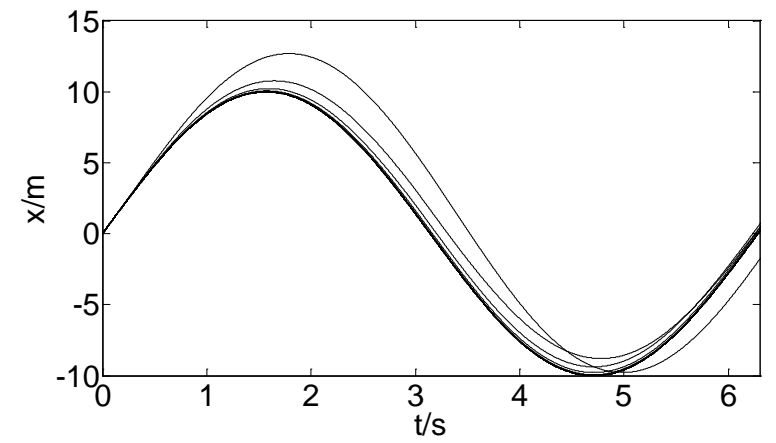

Fig. 3 Position tracking curve of $x$ axis

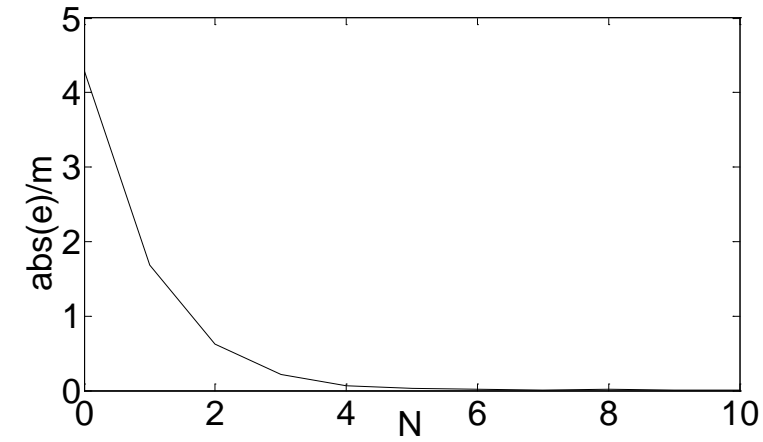

Fig. 4 Position tracking error curve of $x$ axis

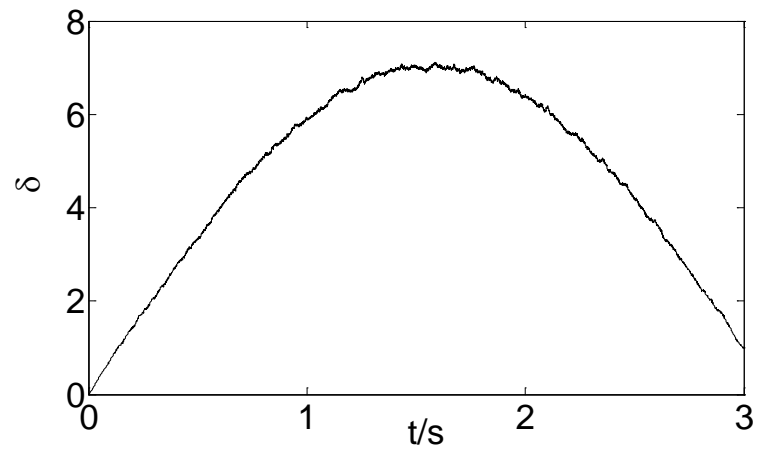

Fig. 5 The change curve of adaptive quantity Delta 


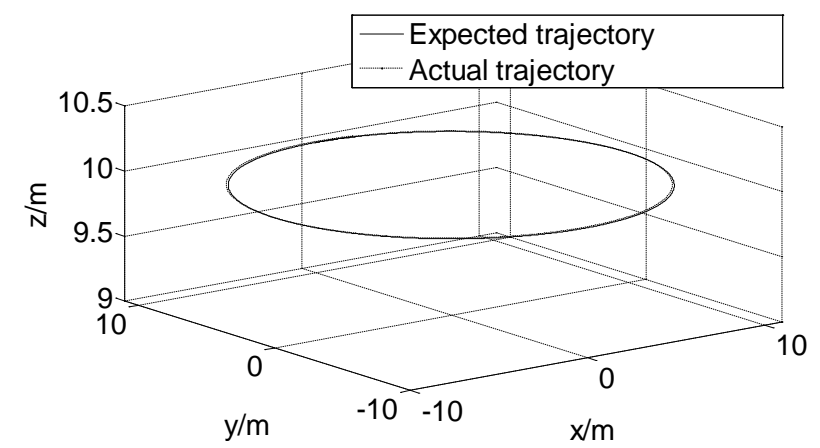

Fig. 6 Horizontal position tracking curve after ten iterations

\section{Conclusion}

Through simulation, we can know that in the case of the exact system model, the algorithm can still achieve the accurate tracking of the trajectory, And has a high ability of anti-interference, The algorithm structure is simple, easy to implement in real system.

\section{References}

[1] Abdelaziz Benallegue,Backstepping Control for a Quadrotor Helicopter[C].Proceedings of the 2006 IEEE/RSJ International Conference on Intelligent Robots and Systems, Beijing,2006: 3225-3260.

[2] Atheer L. Salih;M. Moghavvemi;Haider A. F. Mohamed;Khalaf Sallom Gaeid,Flight PID Controller Design for a UAV Quadrotor[J]. Scientific Research and Essays, 2010,5(23) : 3660-3665.

[3] Rios-Gutierrez ;Kshatri , Fuzzy Controller for a Semi-Autonomous Quad-Rotor Helicopter [J].Computers in Education Journal. 2010,20(1) : 45-60.

[4] Bouabdallah S, Noth A, Sicgwabt R ,PID vs LQ control techniques applied to an indoor micro quadrotor [C].Proceedings of IEEE International Conference on IROS,Sendal, Japan ,2004: 2451-2456.

[5] Wang Shugang. Research of quadrotor control[M]Harbin:Harbin Institute of Technology.2006

[6] Altug, E., Osreiwski, J.O., Mahony, R.: Control of a quadrotor helicopter using visual feedback. [C]. Proceedings of IEEE. International Conference on Robotics and Automation, Washington, DC, 2002: 72-77.

[7] Lee, Daewon ;Jin Kim, H. ;Sastry, Shankar, Feedback linearization vs. adaptive sliding mode control for a quadrotor helicopter[J].International Journal of Control Automation and Systems,2009,7(3) : 419-428.

[8] BOUABDALLAH S, SIEGWART R. Backstepping and sliding-mode techniques applied to an indoor micro quadrotro[C]Proceedings of the 2005 IEEE/RSJ International Conference on Robotics and Automation,2005: 2247-2252.

[9] ASHFAQ A M, WANG Daobo. Modeling and backstepping based nonlinear control strategy for a 6 DOF Quadrotor helicopter[J].Chinese Journal of Aeronautics, 2008, 21(3): 261-268.

[10] Yinqiu Wang;Qinghe Wu;Yao Wang. Distributed cooperative control for multiple quadrotor systems via dynamic surface control.[J].Nonlinear Dynamics,2014,75(3) : 513-527.

[11]Wang Lu,Li Guangchun,Wang Zhaolong,et al.Sliding mode control of an underactuated 
Quadrotor UAV[J]. Journal of Harbin Engineering University.2012.33(10) : 1248-1253.

[12]Bouadi H,Bouchoucha M,Tadjine M. Sliding mode control based on backstepping approach for an UAV type quadrotor[J].International Journal of Applied Mathematics and Computer Sciences, 2008, 4(1): 12-17. 